\title{
Competition and quality in regulated markets: a differential-game approach
}

\author{
Kurt R. Brekke* Roberto Cellini ${ }^{\dagger} \quad$ Luigi Siciliani ${ }^{\ddagger} \quad$ Odd Rune Straume ${ }^{\S}$
}

February 28, 2008

\begin{abstract}
We investigate the effect of competition on quality in regulated markets (e.g., health care, higher education, public utilities) taking a differential game approach, in which quality is a stock variable. Using a Hotelling framework, we derive the open-loop solution (providers commit to an optimal investment plan at the initial period) and the closed-loop solution (providers move investments in response to the dynamics of the states). If the marginal provision cost is constant, the open-loop and closed-loop solutions coincide, implying that static models are robust to a dynamic specification. If the marginal provision cost is increasing, investment and quality are lower in the closed-loop solution: in fact, quality drops to the minimum level in steady state, implying that quality competition is effectively eliminated. In this case, static models tend to exaggerate the positive effect of competition on quality. Our results can explain the mixed empirical evidence on competition and quality for regulated markets.
\end{abstract}

Keywords: Regulated markets; Competition; Quality.

JEL: H42; I11; I18; L13

\footnotetext{
*Department of Economics and Health Economics Bergen, Norwegian School of Economics and Business Administration, Helleveien 30, N-5045 Bergen, Norway. E-mail: kurt.brekke@nhh.no.

${ }^{\dagger}$ Department of Economics, University of Catania, Corso Italia 55 -I95129 Catania, Italy. E-mail: cellini@unict.it.

${ }^{\ddagger}$ Department of Economics and Related Studies, and Centre for Health Economics, University of York, Heslington, York YO10 5DD, UK; and C.E.P.R., 90-98 Goswell Street, London EC1V 7DB, UK. E-mail: ls24@york.ac.uk.

${ }^{\S}$ Department of Economics and NIPE, University of Minho, Campus de Gualtar, 4710-057 Braga, Portugal; and Health Economics Bergen, Norway. E-mail: o.r.straume@eeg.uminho.pt.
} 


\section{Introduction}

Quality is a major concern in many regulated markets, like health care, education and public utilities. In recent years, many highly regulated sectors have been subject to reforms introducing competition, at least along some dimensions. For example, in the health care sector, the combination of prospective payment systems and hospital choice aims at giving hospitals incentives to attract patients (and thus payments) by improving their quality. Similarly, in the (higher) education sector, school choice combined with payments based on the number of students, is perceived to give universities and other educational institutions incentives to compete in terms of (teaching/research) quality in order to attract students and, in turn, revenues.

Most of the existing theoretical literature on quality competition under price regulation reports a positive relationship between competition - measured either as a switch from monopoly to (imperfect) competition or as an increase in the degree of competition intensity - and quality (see, e.g., Ma and Burgess, 1993; Wolinsky, 1997; Brekke et al., 2006; Matsumura and Matsushima, 2007). Similar predictions are obtained in the health care literature (see, e.g., Calem and Rizzo, 1995; Gravelle, 1999; Lyon, 1999; Gravelle and Masiero, 2000; Beitia, 2003; Brekke et al., 2007; Nuscheler, 2003; Karlsson, 2007).

The empirical evidence seems, however, to be more ambiguous. For example, regarding the effect of competition on quality in the health care sector (with fixed prices), Kessler and McClellan (2000) and Tay (2003) find a positive effect, Gowrisankaran and Town (2003) find a negative effect, Shen (2003) finds mixed effects, while Shortell and Hughes (1988) and Mukamel et al. (2001) find no effects. ${ }^{1,2}$

In the present paper we revisit the modelling of quality competition in order to shed some light on the apparent divergence between theory and evidence.

In most of the theoretical literature an important assumption is that quality can be adjusted instantaneously and permanently (at some costs). This is obviously a simplifying assumption

\footnotetext{
${ }^{1}$ See Gaynor (2006) for a survey of theoretical and empirical literature on the relationship between hospital competition and quality. See Lambertini (2006) for theoretical analyses of investment for quality in industrial organization.

${ }^{2}$ For empirical studies of competition and quality in the education sector, see, e.g., Dee (1998), Epple and Romano (1998) and Hoxby (2000).
} 
that may be restrictive. A provider who wants to increase quality will have to invest in it. For example, in the health care sector a hospital will have to train existing doctor, hire more qualified doctors, buy new high-tech equipment and so on. Thus, there might be potentially important implications of the dynamic nature of quality investments that are ignored in a static analysis.

We extend the above mentioned studies by modelling quality as a stock and by adopting a dynamic approach. We develop a model of competition within a Hotelling framework, with two horizontally differentiated providers that potentially differ also with respect to the quality of the good they provide. We assume that quality is a stock that can be increased only if the investment in quality is higher than its deterioration rate. Modelling quality as a stock introduces a dynamic element into the analysis, which turns the problem into a capital accumulation game (Dockner et al., 2000).

We use a differential-game approach to derive the equilibrium quality, under two different behaviour rules followed by providers: in the first setting, providers decide their optimal dynamic plans at the initial period and then stick to it forever (open-loop solution); in the second setting, providers do not commit to an optimal path and set their controls at any time in response to the current value of states (feedback closed-loop solution). The first solution concept might be appropriate when providers can commit to investment plans and stick to it for long periods of time. The second one is appropriate when providers do not or cannot commit to investment plans, but rather observe quality in each period and base their investments on this information.

We find that if the marginal cost of provision is constant, the open-loop and closed-loop solutions coincide: investment and quality are identical under the two solution concepts. This result holds for a general specification of the cost function (cost of quality is either zero, linear or quadratic in quality; investment and quality are either substitutes or complements in costs). We find, as is intuitive, that if the price is above marginal cost, a higher regulated price or lower travel costs (i.e., more competition) increase investment, and thus quality, in steady state. Cost complementarity (substitutability) between investment and quality will increase (decrease) the steady state levels of both. A higher time preference discount rate reduces quality and 
investment under weak regularity conditions, while a higher depreciation rate reduces quality but has an indeterminate effect on investment.

Similar results are obtained under the open-loop solution when we assume that the marginal cost of provision is increasing. However, in contrast to the case with constant marginal cost, investment and quality are lower under the closed-loop solution than under the open-loop solution. In fact, we show that quality drops to the minimum level under the closed-loop solution. Therefore, our model predicts that when (i) quality investment is costly, (ii) the marginal cost is increasing and (iii) providers do not commit to investment plans, then the beneficial effects from competition in terms of higher quality are lower than expected from existing theoretical literature. Indeed, under these conditions, quality competition is effectively eliminated in steady state.

The intuition for this result is related to the fact that, when the marginal cost of provision is increasing, quality investments are strategic complements. In a static setting, this yields strong incentives for quality competition. However, in a dynamic setting, if the players use closed-loop investment rules, lower quality investment by one provider will induce a future reduction in quality investment by the other provider. Thus, due to these strategic dynamics, the players are able to obtain a more collusive outcome in steady state, compared with the open-loop solution or with the solution of a corresponding one-shot game. From the viewpoint of each player, the instantaneous demand loss due to lower quality investments is weighed against the future gains due to the strategic response - lower investment - by the competitor. With an infinite time horizon, as long as the players value future profits, the latter consideration dominates and the closed-loop solution predicts a minimum level of quality in steady state.

The crucial assumption for the above described dynamics is that the marginal cost of provision is increasing. If, on the other hand, marginal production costs are constant, there is no longer any strategic interaction between the players in terms of quality investments, since a fixed price and constant marginal costs imply that marginal revenue is also constant. This explains why the open- and closed-loop solutions coincide in this particular case. Even if the players are able to update their investment plans according to the evolution of quality states, the absence 
of strategic interaction implies that the each player's optimal investment rule does not depend on the choices made by the other player.

In sum, it is clear that our results based on a dynamic analysis partly challenge - in some cases considerably so - the received theoretical literature on competition and quality based on static models, and we believe that our analysis potentially helps explaining some ambiguous evidence. It should also be noted that our results are in contrast with the results obtained within capital accumulation models with a Cournot framework (Dockner, 1992; Dockner et al., 2000). In these models, the providers compete a la Cournot but face capacity constraints that can be relaxed by capital accumulation through investment. It turns out that the investment under the closed-loop solution is in this case higher than under the open-loop one: the more intense competition under the closed-loop solution generates an additional incentive to invest. In contrast, within the regulated markets described in our model, the investment in quality under the closed-loop solution is lower than under the open-loop one.

As previously indicated, our analysis has obvious relevance for health care and education markets, which are heavily regulated in most countries. In hospital markets that are open to free patient choice, hospitals often face fixed treatment prices - usually paid by a third-party payer - and compete for patients in terms of quality of care. With respect to education markets, in many countries universities are predominantly funded by a third-party payer according to student numbers. With a limited possibility of using tuition fees - which are either prohibited or regulated in most European countries - universities have to compete for students in terms of the educational quality they offer (see Brekke, Nuscheler and Straume, 2006, for further discussion). However, the model can also be interpreted in the context of non-price competition in public utilities industries (see Wolinsky, 1997, for examples and discussion). For this particular interpretation, notice that it does not matter, analytically, whether the price of the good is paid by the consumer or by a third-party payer, as long as prices are fixed (regulated). Finally, the model might also be interpreted in the context of pharmaceutical markets. In most countries prices of prescription drugs are regulated and pharmaceutical companies use a considerable amount of resources on marketing in order to capture market shares. Although quality competi- 
tion is not directly relevant in such markets, drug marketing and quality investments share the same essential feature, namely to increase consumers' willingness-to-pay for the good. ${ }^{3}$ Thus, it is possible to reinterpret the quality variable in our model as a marketing variable.

The rest of the paper is organised as follows. The main assumptions of the model are presented in Section 2. Section 3 derives and characterises the equilibrium quality under the open-loop solution, while Section 4 derives and characterises the feedback closed-loop solution. Section 5 concludes the paper.

\section{Model}

In order to analyse the impact of competition on quality, we use a Hotelling framework (Hotelling, 1929). For the sake of concreteness, we will refer to the different variables and parameters of the model in terms of a health care market. ${ }^{4}$

Consider a market for medical treatment with two hospitals located at either end of the unit line $S=[0,1]$. On this line segment there is a uniform distribution of patients, with total mass normalised to 1 . We assume unit demand, where each patient needs - and demands - one hospital treatment in order to be cured. Assuming full market coverage, the decision patients make is to choose which provider to demand treatment from. The utility of a patient who is located at $x \in S$ and seeking treatment at hospital $i$, located at $z_{i}$, is given by ${ }^{5}$

$$
U\left(x, z_{i}\right)=v+k q_{i}-\tau\left|x-z_{i}\right|
$$

where $v$ is the gross valuation of medical treatment, $q_{i} \geq \underline{q}$ is the quality at hospital $i, k$ is a parameter measuring the (marginal) utility of quality, and $\tau$ is a travelling cost parameter. ${ }^{6}$

\footnotetext{
${ }^{3}$ In markets for prescription drugs, marketing activities are also directed towards prescribing physicians in an attempt to increase their "willingness-to-prescribe" (see, e.g., Brekke and Kuhn, 2006; Königbauer, 2007).

${ }^{4}$ We could also specify the model for the market of higher education by replacing hospitals with universities and patients with students. For similar models applied to higher education, see, e.g., Del Rey (2001), De Fraja and Iossa (2002) and De Fraja and Landeras (2006).

${ }^{5}$ There is strong empirical evidence showing that distance and quality are main predictors of patients' choice of hospital, see, e.g., Kessler and McClellan (2000) and Tay (2003).

${ }^{6}$ We refer to distance in physical terms. However, one could also interpret the horizontal dimension in a disease space, where the location of a patient is associated with the disease she suffers from, and the two hospitals are differentiated with respect to the disease they are best able to cure, reflecting hospital specialisation or "service
} 
The lower bound $\underline{q}$ on hospital quality represents the minimum treatment quality hospitals are allowed to offer, implying that $q<q$ can be interpreted as malpractice. For simplicity, we set $\underline{q}=0$. Moreover, we normalise the marginal utility of quality to one, i.e., $k=1$, without of loss of generality. This implies that $\tau$ can be interpreted as the marginal disutility of travelling relative to quality. Thus, a low (high) $\tau$ means that quality is of relatively more (less) importance to the patient than travelling distance.

Since the distance between hospitals is equal to one, the patient who is indifferent between seeking treatment at hospital $i$ and hospital $j$ is located at $x_{i}^{D}$, given by

$$
v+\tau x_{i}^{D}+q_{i}=v-\tau\left(1-x_{i}^{D}\right)+q_{j}
$$

yielding

$$
x_{i}^{D}=\frac{1}{2}+\frac{q_{i}-q_{j}}{2 \tau}
$$

which is also the demand for medical treatment at hospital $i$, given the assumptions of uniform patient distribution (with mass 1) and full market coverage. The provider with a higher quality gets a market share which is more than half. Notice how lower travelling costs make it less costly for patients to switch between hospitals, increasing the demand responsiveness to changes in quality.

Most of the literature assumes that quality can be increased instantaneously and permanently (at some costs). This is, obviously, a simplifying assumption. Quality is a stock which increases when the investment in quality is larger than the depreciation rate of the quality stock. In the hospital context, there are many examples of quality-enhancing investments. For instance, the purchase of MR-machines and CT-scanners improve diagnosing, which in turn will increase the treatment quality. Hospitals invest also in human capital in order to improve quality: they spend money on training of their medical staff, they hire highly skilled physicians (specialists) and nurses, etc. Finally, hospitals invest in facilities to improve, say, the quality of theaters, rooms, catering, etc. ${ }^{7}$

mix" (see, e.g., Calem and Rizzo, 1995, and Brekke et al., 2007).

${ }^{7}$ Some investments might not just influence quality but also capacity. For instance, a new MR-machine might 
Define $I(t)$ as the investment in quality at time $t$, and $\delta$ as the depreciation rate of the quality stock. Analytically, the law of motion of quality is given by:

$$
\frac{d q_{i}(t)}{d t} \equiv \dot{q}_{i}(t)=I_{i}(t)-\delta q_{i}(t)
$$

where $\delta$ is the depreciation rate. Such an accumulation law, à la Solow, is widely used in industrial organization theoretical models to describe capacity accumulation (see Dockner et al., 2000, or Cellini and Lambertini, 2003, for literature reviews).

We assume that hospitals maximise profits. ${ }^{8}$ The instantaneous objective function of hospital $i$ is assumed to be given by

$$
\pi_{i}(t)=T+p x_{i}^{D}\left(q_{i}(t), q_{j}(t)\right)-C\left(I_{i}(t), x_{i}^{D}\left(q_{i}(t), q_{j}(t)\right), q_{i}(t)\right)-F
$$

where $p$ is a regulated price per treatment (patient) price and $T$ is a potential lump-sum transfer received from a third-party payer (insurer). ${ }^{9,10}$

On the cost-side, each hospital $i$ faces a fixed cost $F$ and variable cost $C(\cdot)$ that depends on the quality investment $I_{i}$ and supply of $x_{i}^{D}$ treatments at quality $q_{i}$. We assume that $C(\cdot)$ is increasing in the investment, $C_{I_{i}}>0$, as investment in quality is obviously costly, at an increasing rate, $C_{I_{i} I_{i}}>0$. We also assume that $C(\cdot)$ is weakly increasing in quality, $C_{q_{i}} \geqslant 0$.

The cost function is also increasing in the number of patients treated, $C_{x_{i}}>0$. Below we will distinguish the cases when the cost of treatment is linear $\left(C_{x_{i} x_{i}}=0\right)$ and when it is convex

improve treatment quality, but also the number of patients that can be treated. Here, we restrict attention to pure quality investments. Capacity accumulation has been analysed in detail in several other papers (Dockner et al, 2000).

${ }^{8}$ At first glance, this assumption may seem inappropriate for public and non-profit hospitals due to their constraints on profit distribution. However, hospitals may add to their reserves the financial surplus obtained. Alternatively, managers may spend the surplus to pursue other objectives: they might increase the physician staff, the range of services, or even increase managerial perks (see e.g., Dranove and White, 1994, De Fraja, 2000, Chalkley and Malcomson, 1998ab). Importantly, the empirical evidence shows little support for different behaviour of for-profit and not-for-profit hospitals (Sloan, 2000), suggesting that profit maximisation is a reasonable assumption.

${ }^{9}$ Alternatively, we could have assumed that the payments are collected directly from the consumers, as for public utilities. It is, however, straightforward to show that this will not change our results.

${ }^{10}$ This payment system is also highly relevant for higher education, especially for European universities and colleges, which receive a fixed payment per student as well as a block grant (see e.g., Kaiser et al., 1992). 
$\left(C_{x_{i} x_{i}}>0\right) .{ }^{11}$ We will show that this assumption has important implications for the results. We also make the simplifying assumption that the cost function is separable in the quantity $x_{i}$, implying that $C_{x_{i} q_{i}}=C_{x_{i} I_{i}}=0 .{ }^{12,13}$

In contrast, we assume that $C_{I_{i} q_{i}} \gtrless 0$. If $C_{I_{i} q_{i}}<0$, then quality and investment are complements and a higher quality reduces the marginal cost of investment. If $C_{I_{i} q_{i}}>0$, then quality and investment are substitutes and a higher quality increases the marginal cost of investment.

Defining $\rho$ as the (constant) preference discount rate, the hospital objective function over the infinite time horizon is

$$
\int_{0}^{+\infty} \pi(t) e^{-\rho t} d t .
$$

In reality, providers may not have an infinite-time horizon, but may have reasonably long finite horizons. If the optimal path does not differ significantly from the solution with a very large but finite horizon, the convenience of working with an infinite-horizon model may be worth the loss of realism (see Léonard and van Long, 1992, p. 285). Also, when doctors or managers retire, they may well be replaced by other doctors and managers with similar utility functions, thus generating an infinite-time horizon.

In this type of dynamic models with strategic interactions - i.e., differential games - there are two main solution concepts: a) open-loop solution, where each hospital knows the initial state of the system and then nothing else, i.e., each hospital knows the initial quality of the other provider, but not in the following periods; b) closed-loop solution, where each hospital knows the initial state of the system, but also later knows the state variable values, i.e., each hospital knows the quality of the other provider, not only in the initial state, but also in all of the subsequent periods. Within the closed-loop solutions, further distinctions can be made: if one assumes that players take into account only the initial state and the current state, the

\footnotetext{
${ }^{11} \mathrm{~A}$ convex variable cost function is normally supported by the evidence suggesting that economies of scale are quite rapidly exhausted in the hospital sector (see, e.g., Ferguson et al., 1999, and Folland et al., 2004, for literature surveys).

${ }^{12}$ The assumption of cost separability between quality and quantity is widely used in the related literature (see, e.g., Economides, 1989, 1993; Calem and Rizzo, 1995; Lyon, 1999; Gravelle and Masiero, 2000; Barros and Martinez-Giralt, 2002).

${ }^{13}$ The assumption of separability between investment and quantity is done to focus on quality dynamics rather than capacity dynamics. As mentioned above, we restrict attention to investments that affect quality only.
} 
"memoryless" closed-loop solution is obtained; if players take into account the whole history of states, the "perfect state" closed-loop is obtained; finally, if players in each instant take into account the current value of states (i.e., the whole past history is summarized by the current value of states), the feedback rule is obtained. Typically, the feedback closed-loop solution is obtained based on the Bellman equation.

In order to establish which one is the most appropriate solution concept, it is essential to evaluate the relevant information set used by players when they take their decisions. In the cases in which the collection of information over time is difficult, it is reasonable to model the choice according to the open-loop rule; on the opposite, when players can observe the current state of the world and they behave accordingly, the closed rules are more appropriate. Clearly, closed-loop solutions are more appealing, but solving for closed-loop is more difficult. However, in some cases - and health care markets can be a good example - players might have to commit to investment plans and stick to them for long periods of time. In this case, the open-loop solution might be the relevant one. Nevertheless, there is a wide range of problems where the two solutions coincide. ${ }^{14}$ Below, we compare the closed-loop and open-loop solutions. Section 3 provides the open-loop solution, while Section 4 provides the closed-loop one, under the specific rule of feedback behaviour.

\section{Open-loop solution}

Provider $i$ 's maximisation problem is given by

$$
\text { Maximise } \int_{0}^{+\infty} \pi_{i}(t) e^{-\rho t} d t,
$$

\footnotetext{
${ }^{14}$ Games where this coincidence arises are presented in Clemhout and Wan (1974); Reinganum (1982); Mehlmann and Willing (1983); Dockner, Feichtinger and Jørgensen (1985). See also Mehlmann (1988), Fershtman, Kamien and Muller (1992), Dockner et al. (2000, ch. 7) for review.
} 


$$
\begin{aligned}
\text { subject to } & \dot{q}_{i}(t)=I_{i}(t)-\delta q_{i}(t), \\
\dot{q}_{j}(t) & =I_{j}(t)-\delta q_{j}(t), \\
q_{i}(0) & =q_{i 0}>0, \\
q_{j}(0) & =q_{j 0}>0 .
\end{aligned}
$$

Let $\mu_{i}(t)$ and $\mu_{j}(t)$ be the current value co-state variables associated with the two state equations. The current-value Hamiltonian is: ${ }^{15}$

$$
H_{i}=T+p x_{i}^{D}\left(q_{i}, q_{j}\right)-C\left(I_{i}, x_{i}^{D}\left(q_{i}, q_{j}\right), q_{i}\right)-F+\mu_{i}\left(I_{i}-\delta q_{i}\right)+\mu_{j}\left(I_{j}-\delta q_{j}\right),
$$

The solution is given by (a) $\partial H_{i} / \partial I_{i}=0$, (b) $\dot{\mu}_{i}=\rho \mu_{i}-\partial H_{i} / \partial q_{i}$, (c) $\partial H_{i} / \partial \mu_{i}=0$, (d) $\dot{\mu}_{j}=\rho \mu_{j}-\partial H_{i} / \partial q_{j}$, or more extensively:

$$
\begin{aligned}
\mu_{i} & =C_{I_{i}} \\
\dot{\mu}_{i} & =-\frac{1}{2 \tau}\left(p-C_{x_{i}}\right)+C_{q_{i}}+\mu_{i}(\delta+\rho), \\
\dot{q}_{i} & =I_{i}-\delta q_{i} \\
\dot{\mu}_{j} & =\frac{p}{2 \tau}+\mu_{j}(\delta+\rho),
\end{aligned}
$$

to be considered along with the transversality condition $\lim _{t \rightarrow+\infty} \mu_{i}(t) q_{i}(t)=0$. The second order conditions are satisfied if the Hamiltonian is concave in the control and state variables (Léonard and Van Long, 1992)..$^{16}$

Totally differentiating the first equation with respect to time we obtain $\dot{\mu}_{i}=C_{I_{i} I_{i}} \dot{I}_{i}+C_{I_{i} q_{i}} \dot{q}_{i}$ or, after substitution, $\dot{\mu}_{i}=C_{I_{i} I_{i}} \dot{I}_{i}+C_{I_{i} q_{i}}\left(I_{i}-\delta q_{i}\right)$. Substituting into the second equation (and using $\mu_{i}=C_{I_{i}}$, we obtain:

$$
\dot{I}_{i}=\frac{-\frac{1}{2 \tau}\left(p-C_{x_{i}}\right)+C_{q_{i}}+(\delta+\rho) C_{I_{i}}-C_{I_{i} q_{i}}\left(I_{i}-\delta q_{i}\right)}{C_{I_{i} I_{i}}},
$$

\footnotetext{
${ }^{15}$ The indication of time $(t)$ is omitted, to ease notation.

${ }^{16}$ This is the case since (a) $H_{I_{i} I_{i}}=-C_{I_{i} I_{i}}<0$; (b) $H_{q_{i} q_{i}}=-C_{q_{i} q_{i}}-\left[1 /(2 \tau)^{2}\right] C_{x_{i} x_{i}}<0$; (c) $H_{I_{i} I_{i}} H_{q_{i} q_{i}}>$ $\left(H_{I_{i} q_{i}}\right)^{2}$ or $C_{I_{i} I_{i}}\left\{C_{q_{i} q_{i}}+\left[1 /(2 \tau)^{2}\right] C_{x_{i} x_{i}}\right\}>C_{I_{i} q_{i}}^{2}$.
} 
which, together with $\dot{q}_{i}=I_{i}-\delta q_{i}$, describe the dynamics of the equilibrium.

Totally differentiating the locus of investment, $\dot{I}_{i}=0$, and rearranging yields

$$
\left.\frac{\partial I_{i}}{\partial q_{i}}\right|_{I_{i}=0}=-\frac{\left(\frac{1}{2 \tau}\right)^{2}\left(C_{x_{i} x_{i}}\right)+C_{q_{i} q_{i}}+(2 \delta+\rho) C_{I_{i} q_{i}}-C_{I_{i} q_{i} q_{i}}\left(I_{i}-\delta q_{i}\right)}{(\delta+\rho) C_{I_{i} I_{i}}-C_{I_{i} I_{i} q_{i}}\left(I_{i}-\delta q_{i}\right)} .
$$

Sufficient but not necessary conditions for the locus of investment, $\dot{I}_{i}=0$, to be negatively sloped are $C_{I_{i} q_{i}}>0$ and $C_{I_{i} q_{i} q_{i}}=C_{I_{i} I_{i} q_{i}}=0$. The second locus is $\dot{q}_{i}=0$, or $I_{i}=\delta q_{i}$, with $\frac{\partial I_{i}}{\partial q_{i}}>0$.

The dynamics of investment and quality can be represented in matrix form as follows:

$$
\begin{aligned}
{\left[\begin{array}{c}
\dot{I}(t) \\
\dot{q}(t)
\end{array}\right]=} & {\left[\begin{array}{cc}
(\delta+\rho)-\frac{C_{I_{i} I_{i} q_{i}}}{C_{I_{i} I_{i}}}\left(I_{i}-\delta q_{i}\right) & {\left[\begin{array}{c}
\left(\frac{1}{2 \tau}\right)^{2}\left(C_{x_{i} x_{i}}\right)+(2 \delta+\rho) C_{I_{i} q_{i}} \\
1
\end{array}\right]} \\
1 & \frac{C_{q_{i} q_{i}}-C_{I_{i} q_{i} q_{i}}\left(I_{i}-\delta q_{i}\right)}{C_{I_{i} I_{i}}}
\end{array}\right] } \\
& +\left[\begin{array}{c}
-(p-c) /(2 \gamma \tau) \\
0
\end{array}\right]
\end{aligned}
$$

where the 2-by-2 matrix is the Jacobian $J$ of the dynamic system. As for the dynamic properties of the system, suppose that this is evaluated around the steady state, or alternatively that third-order derivatives of the cost function are set to zero: $C_{I_{i} I_{i} q_{i}}=C_{I_{i} q_{i} q_{i}}=0$. Then, it is immediate to check that the Jacobian matrix $J$ in (19) is such that $\operatorname{tr}(J)=\rho>0$, and $\operatorname{det}(J)=-\delta(\delta+\rho)-\frac{\left(\frac{1}{2 \tau}\right)^{2}\left(C_{x_{i} x_{i}}\right)+C_{q_{i} q_{i}}+(2 \delta+\rho) C_{I_{i} q_{i}}}{C_{I_{i} I_{i}}}<0$, implying that the equilibrium is stable in the saddle sense. The solution is described in Figure 1.

[Figure 1 about here]

Let $q^{s}$ be the steady state level of quality and suppose we start off equilibrium, at level $q_{0}<q^{s}$. The solution is then characterised by a period of increasing quality and decreasing investment in quality. Suppose instead that $q_{0}>q^{s}$. In this case, we should observe a period of decreasing quality and increasing investment. Notice how off equilibrium, investment and quality 
move over time in opposite directions while in the steady state investment is proportional to quality $\left(I_{i}=\delta q_{i}\right)$.

In Figure 1 we have assumed the investment locus, $\dot{I}_{i}=0$, to be negatively sloped , implying that the equilibrium is a saddle point. The equilibrium can still be a saddle point also when $\dot{I}_{i}=0$ is positively sloped, as long as it is not too positively sloped. This case arises, for example, when the marginal cost of quality and the marginal cost of provision are constant but quality and investment are substitutes, which implies $C_{I_{i} q_{i}}<0$. The equilibrium is still a saddle point if $\operatorname{det}(J)=-\delta(\delta+\rho)-\frac{(2 \delta+\rho) C_{I_{i} q_{i}}}{C_{I_{i} I_{i}}}<0 .{ }^{17}$

Figure 2 shows some comparative dynamics. Suppose the system is in an initial steady state. Figure 2 shows the effect of an unexpected increase in price or reduction in travel costs (more competition) on investment and quality. The locus $\dot{I}_{i}=0$ shifts upwards. The shock generates a positive jump in investment (overshooting), and it is followed by a period of decreasing investment and increasing quality.

[Figure 2 about here]

In the following, we will consider two special cases: constant and increasing marginal cost of treatment. Later on, the specification of the treatment cost function will be shown to have crucial implications with respect to the comparison of the open-loop and closed-loop solutions.

\subsection{Constant marginal treatment cost}

Suppose that marginal cost of treatment is constant, while it is quadratic in investment and quality:

$$
C\left(I_{i}, x_{i}^{D}, q_{i}\right)=c x_{i}^{D}+\frac{\gamma}{2} I_{i}^{2}+\frac{\beta}{2} q_{i}^{2}+\varphi q_{i} I_{i}
$$

With this specification, the solution of the Hamiltonian system leads to

$$
\dot{I}_{i}=-\frac{1}{2 \tau \gamma}(p-c)+(\delta+\rho) I_{i}+\frac{1}{\gamma}[\beta+(2 \delta+\rho) \varphi] q_{i} .
$$

\footnotetext{
${ }^{17}$ If the determinant is positive, then we have an unstable node.
} 
In the steady state we have $\dot{q}_{i}=0$, implying $I_{i}=\delta q_{i}$, which, substituted into $\dot{I}_{i}=0$, yields

$$
I_{O L}^{S}=\frac{(p-c) \delta}{2 \tau[\beta+\gamma \delta(\delta+\rho)+\varphi(2 \delta+\rho)]}
$$

and

$$
q_{O L}^{s}=\frac{I_{O L}^{s}}{\delta},
$$

where superscript $s$ and subscript $O L$ indicate the steady state levels in the open-loop solution, respectively.

The results are reasonable. If the price is above the marginal treatment cost, then lower travel $\operatorname{costs}(\tau)$ or a higher price $(p)$ increase quality and investment. Similarly, a higher marginal cost of quality $(\beta)$ or of investment $(\gamma)$ reduce quality and investment. If quality and investment are complements $(\varphi>0)$, then quality and investment are higher in the steady state; if they are substitutes, quality and investment are lower in the steady state. A higher time preference discount rate $(\rho)$ reduces quality and investment whenever $\gamma \delta+\varphi>0 .{ }^{18}$ Notice also how a higher depreciation rate of quality $(\delta)$ is associated with lower steady state quality, while the effect on investment is indeterminate. ${ }^{19}$

\subsection{Increasing marginal treatment cost}

Suppose that marginal cost of treatment is increasing, while it is still quadratic in investment and quality:

$$
C\left(I_{i}, x_{i}^{D}, q_{i}\right)=\frac{c}{2}\left(x_{i}^{D}\right)^{2}+\frac{\gamma}{2} I_{i}^{2}+\frac{\beta}{2} q_{i}^{2}+\varphi q_{i} I_{i} .
$$

\footnotetext{
${ }^{18}>$ From $(22)$ we obtain$$
\frac{\partial I_{O L}^{s}}{\partial \rho}=-\frac{(p-c) \delta(\gamma \delta+\varphi)}{2 \tau[\beta+\gamma \delta(\delta+\rho)+\varphi(2 \delta+\rho)]^{2}}
$$

${ }^{19}>$ From (22) we obtain

$$
\frac{\partial I_{O L}^{s}}{\partial \delta}=\frac{(p-c)\left(\rho \varphi+\beta-\gamma \delta^{2}\right)}{2 \tau[\beta+\varphi(2 \delta+\rho)+\delta \gamma(\delta+\rho)]^{2}} .
$$

The sign of this expression is determined by the sign of $\left(\rho \varphi+\beta-\gamma \delta^{2}\right)$, which is indeterminate.
} 
With this particular specification, the solution of the Hamiltonian system yields

$$
\dot{I}_{i}=-\frac{1}{2 \tau \gamma}\left(p-c\left(\frac{1}{2}+\frac{q_{i}-q_{j}}{2 \tau}\right)\right)+\frac{\beta}{\gamma} q_{i}+(\delta+\rho) I_{i}+\frac{\varphi}{\gamma} q_{i}(2 \delta+\rho) .
$$

The symmetric steady state equilibrium, $\dot{I}_{i}=0$ and $\dot{q}_{i}=0$, together with $I_{i}=\delta q_{i}$, yield

$$
I_{O L}^{S}=\frac{\left(p-\frac{c}{2}\right) \delta}{2 \tau[\beta+(\delta+\rho) \gamma \delta+\varphi(2 \delta+\rho)]}
$$

and

$$
q_{O L}^{s}=\frac{I_{O L}^{s}}{\delta}
$$

which is similar to the solution with constant marginal cost, and requires no further comments.

\section{Closed-loop solution}

The open-loop rule is realistic in cases where the players compute their investment plans at the beginning, and then stick to them forever. In other cases, however, players take their decisions while observing the evolution of states, and the relevant rule is then of the closed-loop type. In this section we derive the so-called feedback rule, where the controls set by the players depend on the current level of states. Open-loop solutions are simpler to compute, but they are only weakly time consistent. On the opposite, closed-loop solutions - which are more involved to compute - are strongly time consistent. There is a wide body of literature on the cases of coincidence between the time path of controls and states under different solution concepts. In the lucky case in which they coincide, the (more easily computable) open-loop solutions are strongly time consistent (see Mehlman, 1982). 


\subsection{Constant marginal treatment cost}

Applying the cost specification in (20), the optimal rule for player $i$ in the closed-loop solution is given by ${ }^{20}$

$$
I_{i}(t)=\phi_{i}^{C L}\left(q_{i}(t), q_{j}(t)\right)=\frac{1}{\gamma}\left[\alpha_{1}+\left(\alpha_{3}-\varphi\right) q_{i}(t)\right]
$$

where

$$
\alpha_{1}=\frac{p-c}{\tau\left[\rho+\sqrt{4 \frac{\beta+\rho \varphi+2 \delta \varphi}{\gamma}+(\rho+2 \delta)^{2}}\right]}>0
$$

and

$$
\alpha_{3}-\varphi=\gamma\left(\frac{\rho}{2}+\delta\right)-\sqrt{\gamma(\beta+\rho \varphi+2 \delta \varphi)+\gamma^{2}\left(\frac{\rho}{2}+\delta\right)^{2}}<0
$$

Using that fact that $I_{i}=\delta q_{i}$ in steady state, it is easily shown that the steady state equilibrium in the closed-loop solution coincides with the previously derived open-loop steady state equilibrium, given by (22)-(23), i.e., $I_{C L}^{s}=I_{O L}^{s}=\delta q_{O L}^{s}=\delta q_{C L}^{s}$. This constitutes the first main result of the paper:

Proposition 1 If the marginal treatment cost is constant, the open-loop and closed-loop solutions coincide in steady state.

The coincidence between the two solutions clearly holds also for the special cases when $\varphi=0$ ( or $\varphi=\beta=0$ ). We can also show that the same result holds when the cost of quality is linear rather than convex (i.e., $C\left(I_{i}, x_{i}^{D}, q_{i}\right)=c x_{i}^{D}+\frac{\gamma}{2} I_{i}^{2}+\beta q_{i}+\varphi q_{i} I_{i}$; the proof is omitted).

The important contributing factor to this coincidence result is that there is a constant marginal (instantaneous) revenue gain of quality investments, implying that the optimal dynamic investment rule for each player, as given by (28), is independent of the quality level provided by the other player. In other words, the optimal investment path for hospital $i$ does not depend on the quality stock of hospital $j$, implying an absence of strategic interaction in this particular respect. Given that the cost function is separable in quantity, this feature - constant marginal revenue - is always present when the treatment price is fixed and the marginal treatment cost is constant. Consequently, our analysis suggests that, if the marginal cost is constant, the solu-

\footnotetext{
${ }^{20}$ See Appendix A for the details of this solution.
} 
tion within a dynamic approach (regardless of the open- or closed-loop solution) is qualitatively similar to the ones that would be obtained within a static approach. We can thus conclude that the static analysis is reasonably robust in this particular case.

\subsection{Increasing marginal treatment cost}

Suppose instead that the marginal treatment cost is increasing, with the cost function given by (24). To keep the analysis simple, we assume that quality does not affect the marginal cost of investment, i.e., $\varphi=0$. As we have shown above, this does not affect the coincidence result in Proposition 1. With this specification, the optimal investment rule for player $i$ is given by ${ }^{21}$

$$
I_{i}(t)=\phi_{i}^{C L}\left(q_{i}(t), q_{j}(t)\right)=\frac{1}{\gamma}\left[\alpha_{1}+\alpha_{3} q_{i}(t)+\alpha_{5} q_{j}(t)\right]
$$

where

$$
\begin{aligned}
& \alpha_{1}=\frac{\left(p-\frac{c}{2}\right)\left(\alpha_{5}-\rho \gamma\right)}{4 \tau[\beta+\gamma \delta(\rho+\delta)]}>0, \\
& \alpha_{3}=\frac{1}{2}\left[\gamma(\rho+2 \delta)-\alpha_{5}\right]<0,
\end{aligned}
$$

and

$$
\alpha_{5}=\frac{\gamma}{3} \sqrt{\frac{1}{\gamma}\left(\frac{c}{\tau^{2}}+4 \beta\right)+(\rho+2 \delta)^{2}}>0
$$

Applying (31)-(34) to the steady state condition $I_{i}=\delta q_{i}$, and keeping in mind that investments must be non-negative, the steady state investment and quality in the closed-loop solution are then given by

$$
I_{C L}^{s}=\max \left\{0,\left(\frac{\rho-\frac{\alpha_{5}}{\gamma}}{\rho+\frac{\alpha_{5}}{\gamma}}\right) I_{O L}^{s}\right\}=0
$$

and

$$
q_{C L}^{s}=\frac{I_{C L}^{s}}{\delta}=0
$$

where $I_{O L}^{s}$ is the steady state level of investment in the open-loop solution with increasing marginal treatment costs, as given by (26) with $\varphi=0$. Thus, since $\alpha_{5}>\rho \gamma$, the closed-loop

\footnotetext{
${ }^{21}$ The derivation of the closed-loop solution with increasing marginal costs is presented in Appendix B.
} 
solution is a corner solution where the steady state levels of investment and quality are at the minimum level. ${ }^{22}$ In other words, dynamic strategic competition between providers using closed-loop investment rules effectively eliminates quality competition in steady state.

Proposition 2 If the marginal treatment cost is increasing, the steady state levels of investment and quality under the closed-loop solution are at the minimum level.

When marginal treatment costs are non-constant, marginal revenue is no longer independent of quality levels, since quality changes will affect demand and, in turn, the marginal treatment cost facing each hospital. This introduces a strategic interaction in the sense that the optimal investment rule for player $i$ depends, at each point in time, on the quality stock of player $j$, as we can see from (31). This dynamic interaction is reflected in the parameter $\alpha_{5}$. More specifically, with increasing marginal costs, quality investments are strategic complements. A quality increase by hospital $i$ will shift demand away from hospital $j$, implying that the marginal cost of hospital $j$ decreases. Since the price is constant, this increases the profit margin of hospital $j$, making quality investments more profitable on the margin for this hospital. Conversely, a quality decrease by hospital $i$ will be strategically followed by a quality decrease by hospital $j$.

This strategic interaction has important implications for the players' dynamic competition incentives. From the perspective of the profit-maximising providers, the business-stealing effect of quality investments constitutes a form of "destructive competition". This is particularly pronounced in the case of inelastic total demand, where quality investments have a purely business-stealing effect. Compared with the outcome of a static game, the open-loop solution in a dynamic setting does not (qualitatively) produce a less competitive outcome, since it has the essential characteristics of a one-shot game, where the players make once-and-for-all commitments to their investment plans at the outset of the game. This is also true for the closed-loop solution in the case of constant marginal costs, due to the aforementioned absence of strategic interaction.

However, the presence of increasing marginal costs introduces a dynamic strategic interaction in terms of quality investments, as explained above. When the players revise their investment

\footnotetext{
${ }^{22}$ Notice that concavity of the value functions require that $\alpha_{3}<0$ (see Appendix B). Using the definition of $\alpha_{5}$, we see that this always holds if $\beta$ is sufficiently large, or if $c$ is sufficiently large relative to $\tau$.
} 
plans according to the evolution of quality states, a decrease in quality investment by hospital $i$ will invoke an investment-reducing response by hospital $j$. From the viewpoint of hospital $i$, the instantaneous loss in market share by reducing the supply of quality is weighed against the future gain of a quality reduction - a strategic response - by hospital $j$. As long as the players value future profits, the dynamic strategic interaction will drive the supply of quality in the market to the minimum level in steady state, effectively eliminating competition in the market.

To summarise the above analysis, our general message is that, in the presence of convex production costs, when dynamic strategic interaction is taken into account, the closed-loop solution indicates that the benefits of competition with respect to quality provision are overestimated in the existing theoretical literature, which is based on static competition models. It is also worth noticing that our main result has a strong implication with respect to regulation policy. A direct implication of Proposition 2 is the following:

Corollary 1 If the marginal treatment cost is increasing, and the providers use closed-loop investment rules, the treatment price $p$ ceases to be an effective policy instrument to stimulate higher quality of treatment.

In other words, and in contrast to the predictions from static models, it might not be possible for a regulator to induce a higher supply of quality by increasing the price. According to our analysis, this will be the case if treatment costs are convex and providers use closed-loop investment rules. In this case, steady state quality is at the minimum level, regardless of $p$, and it is not possible to get out of the corner solution by simple price regulation. The reason for this negative result is that, although the short-run incentives for quality competition increases, the long-run gain of avoiding quality competition increases correspondingly, leaving the players' strategic incentives unaffected in a dynamic setting.

\section{Conclusions}

We have investigated the effect of competition on quality in regulated markets within a Hotelling framework. Differently from the existing literature we assume that quality cannot adjust instan- 
taneously, but rather is a stock variable which increases when investment in quality is higher than the depreciation rate of quality. We investigate the optimal open-loop solution (when providers commit to an optimal plan of investment at the initial period) and the equilibrium closed-loop solution (under the feedback rule, where the providers move their investment in response to the dynamics of the states).

We find that if the marginal cost of provision is constant, the open-loop and closed-loop solution coincide: investment and quality are identical under the two solution concepts. This result suggests that previous predictions obtained from static models are robust to a dynamic specification. For example, if the price is above the marginal cost, a higher regulated price or lower travel costs (i.e., more competition) increase quality and investment. Moreover, we show (differently from static analyses) that a higher time preference discount rate reduces quality and investment under weak regularity conditions. Also, a higher depreciation rate reduces quality but has an indeterminate effect on investment.

However, if we assume that the marginal cost is increasing, then the open-loop and closedloop solution do not coincide, which implies that the main results from closed-loop solutions departs from the predictions of static models. Investment and quality are lower under the closedloop solution than under the open-loop solution: in the closed-loop solution, quality drops to the minimum level in steady state. Therefore, our model predicts that the beneficial effects from competition in terms of higher quality are lower than expected from existing theoretical literature. 


\section{References}

[1] Barros, P.P., Martinez-Giralt, X., 2002. Public and private provision of health care. Journal of Economics \& Management Strategy, 11, 109-133.

[2] Başar, T., Olsder, G.J. 1982, 1995². Dynamic Noncooperative Game Theory, San Diego, Academic Press.

[3] Beitia, A., 2003. Hospital quality choice and market structure in a regulated duopoly. Journal of Health Economics, 22, 1011-1036.

[4] Brekke, K.R., Kuhn, M., 2006. Direct to consumer advertising in pharmaceutical markets. Journal of Health Economics, 25, 102-130.

[5] Brekke, K.R., Nuscheler, R., Straume, O.R., 2006. Quality and location choices under price regulation. Journal of Economics \& Management Strategy, 15, 207-227.

[6] Brekke, K.R., Nuscheler, R., Straume, O.R., 2007. Gatekeeping in health care. Journal of Health Economics, 26, 149-170.

[7] Calem, P.S., Rizzo, J.A., 1995. Competition and specialization in the hospital industry: an application of Hotelling's location model. Southern Economic Journal, 61, 1182-1198.

[8] Cellini, R., Lambertini, L., 2003. "Differential oligopoly games", in P. Bianchi and L. Lambertini (eds), Technology, information and market dynamics: Topics in advanced industrial organization, Cheltenham, Edward Elgar, 173-207.

[9] Chalkley, M., Malcomson, J.M., 1998a. Contracting for health services with unmonitored quality. Economic Journal, 108, 1093-1110.

[10] Chalkley, M., Malcomson, J.M., 1998b. Contracting for health services when patient demand does not reflect quality. Journal of Health Economics 17, 1-19.

[11] Clemhout, S., Wan Jr., H.Y., 1974. A class of trilinear differential games. Journal of Optimization Theory and Applications, 14, 419-24. 
[12] De Fraja, G., 2000. Contracts for health care and asymmetric information. Journal of Health Economics, 19, 663-677.

[13] De Fraja G., Iossa, E., 2002. Competition among universities and the emergence of the elite institution. Bulletin of Economic Research 54, 275-293.

[14] De Fraja G., Landeras, P., 2006. Could do better: the effectiveness of incentives and competition in schools. Journal of Public Economics 90, 189-213.

[15] Dee, T.S., 1998. Competition and the quality of public schools. Economics of Education Review 17, 419-427.

[16] Del Rey, E., 2001. Teaching versus research: a model of state university competition. Journal of Urban Economics 49, 356-373.

[17] Dockner, E.J., 1992. A dynamic theory of conjectural variations. Journal of Industrial Economics, 40, 377-395.

[18] Dockner, E.J., Feichtinger, G., Jørgensen, S., 1985. Tractable classes of non-zerosum openloop Nash differential games: Theory and examples. Journal of Optimization Theory and Applications, 45, 179-197.

[19] Dockner, E.J., Jørgensen, S., Van Long, N., Sorger, G., 2000. Differential games in economics and management science. Cambridge: Cambridge University Press.

[20] Dranove, D., White, W.D., 1994. Recent theory and evidence on competition in hospital markets. Journal of Economics and Management Strategy, 3, 169- 209.

[21] Economides, N., 1989. Quality variations and maximal variety differentiation. Regional Science and Urban Economics, 19, 21-29.

[22] Economides, N., 1993. Quality variations in the circular model of variety-differentiated products. Regional Science and Urban Economics, 23, 235-257.

[23] Epple, D., Romano, R.E., 1998. Competition between private and public schools, vouchers and peer group effects. American Economic Review 88, 33-62. 
[24] Ferguson, B., Sheldon, T., Posnett, J. (eds.), 1999. Concentration and choice in health care. London: Royal Society of Medicine.

[25] Fershtman, C., 1987. Identification of classes of differential games for which the open-loop is a degenerate feedback Nash equilibrium. Journal of Optimization Theory and Applications, $55,217-31$.

[26] Fershtman, C., M. Kamien, Muller, E., 1992. "Integral games: Theory and applications", in Feichtinger, G. (ed.), Dynamic economic models and optimal control, Amsterdam, NorthHolland, 297-311.

[27] Folland, S., Goodman, A.C., Stano, M., 2004. The economics of health and health care. Upper Saddle River, NJ: Prentice Hall.

[28] Gaynor, M., 2006. What do we know about competition and quality in health care markets? Foundations and Trends in Microeconomics, Vol. 2, Issue 6.

[29] Gowrisankaran, G., Town, R., 2003. Competition, payers, and hospital quality. Health Services Research, 38, 1403-1422.

[30] Gravelle, H., 1999. Capitation contracts: access and quality. Journal of Health Economics, $18,3,315-340$.

[31] Gravelle, H., Masiero, G., 2000. Quality incentives in a regulated market with imperfect competition and switching costs: capitation in general practice. Journal of Health Economics, 19, 1067-1088.

[32] Hotelling, 1929. Stability in competition. Economic Journal, 39, 41-57.

[33] Hoxby, C.M., 2000. Does competition among public schools benefit students and taxpayers? American Economic Review 90, 1209-1238.

[34] Karlsson, M., 2007. Quality incentives for GPs in a regulated market. Journal of Health Economics, 26, 699-720. 
[35] Kessler, D., McClellan, M., 2000. Is hospital competition socially wasteful? Quarterly Journal of Economics, 115, 577-615.

[36] Königbauer, I., 2007. Advertising and generic market entry. Journal of Health Economics, $26,286-305$.

[37] Lambertini, L. 2006. The economics of vertically differentiated markets. Cheltenham, Edward Elgar.

[38] Léonard, D., Van Long, N., 1992. Optimal control theory and static optimization in economics. Cambridge: Cambridge University Press.

[39] Lyon, T.P., 1999. Quality competition, insurance, and consumer choice in health care markets. Journal of Economics \& Management Strategy, 8, 545-580.

[40] Ma, C.A., Burgess, J.F., 1993. Quality competition, welfare, and regulation. Journal of Economics, 58, 153-173.

[41] Matsumura, T., Matsushima, N., 2007. Congestion-reducing investments and economic welfare in a Hotelling model. Economics Letters, 96, 161-167.

[42] Mehlmann, A., Willing, R., 1983. On nonunique closed-loop Nash equilibria for a class of differential games with a unique and degenerate feedback solution. Journal of Optimization Theory and Applications, 41, 463-472.

[43] Mehlmann, A. 1988. Applied differential games. New York, Plenum Press.

[44] Mukamel, D., Zwanziger, J., Tomaszewski, K.J., 2001. HMO penetration, competition and risk-adjusted hospital mortality. Health Services Research, 36, 1019-1035.

[45] Nuscheler, R., 2003. Physician reimbursement, time-consistency and the quality of care. Journal of Institutional and Theoretical Economics, 159, 302-322.

[46] Reinganum, J.F., 1982. A class of differential games for which the closed-loop and open-loop equilibria coincide. Journal of Optimization Theory and Applications, 36, 253-262. 
[47] Shen, Y.-S., 2003. The effect of financial pressure on the quality of care in hospitals. Journal of Health Economics, 22, 243-269.

[48] Shortell, S.M., Hughes, E.F., 1988. The effects of competition, regulation, and ownership on mortality rates among hospital inpatients. New England Journal of Medicine, 318, 11001107.

[49] Sloan, F. A., 2000. "Not-for-profit ownership and hospital behavior." In: A. J. Culyer and J. P. Newhouse (eds.), Handbook of Health Economics, Amsterdam: Elsevier, Chapter 21.

[50] Tay, A., 2003. Assessing competition in hospital care markets: the importance of accounting for quality differentiation. RAND Journal of Economics, 34, 786-814.

[51] Wolinsky, A., 1997. Regulation of duopoly: managed competition vs. regulated monopolies. Journal of Economics and Management Strategy, 6, 821-847. 


\section{Appendix A. Closed-loop solution with constant marginal cost}

We assume that the marginal cost of treatment is constant, while it is quadratic in investment and quality so that $C\left(I_{i}, x_{i}^{D}, q_{i}\right)=c x_{i}^{D}+\frac{\gamma}{2} I_{i}^{2}+\frac{\beta}{2} q_{i}^{2}+\varphi q_{i} I_{i}$. The purchaser instantaneous objective function is

$$
T+(p-c)\left(\frac{1}{2}+\frac{q_{i}-q_{j}}{2 \tau}\right)-\frac{\gamma}{2} I_{i}^{2}-\frac{\beta}{2} q_{i}^{2}-\varphi q_{i} I_{i}
$$

which - faced with the linear dynamic constraint - gives rise to a linear-quadratic problem. Hence, define the value function as

$$
V^{i}\left(q_{i}, q_{j}\right)=\alpha_{0}+\alpha_{1} q_{i}+\alpha_{2} q_{j}+\left(\alpha_{3} / 2\right) q_{i}^{2}+\left(\alpha_{4} / 2\right) q_{j}^{2}+\alpha_{5} q_{i} q_{j}
$$

Define $I_{i}=\phi_{i}\left(q_{i}, q_{j}\right)$ and $I_{j}=\phi_{j}\left(q_{i}, q_{j}\right)$. The value function has to satisfy the Hamilton-JacobiBellman (HJB) equation:

$$
\rho V^{i}\left(q_{i}, q_{j}\right)=\max \left\{\begin{array}{c}
T+(p-c)\left(\frac{1}{2}+\frac{q_{i}-q_{j}}{2 \tau}\right)-\frac{\gamma}{2} I_{i}^{2}-\frac{\beta}{2} q_{i}^{2}-\varphi q_{i} I_{i} \\
+V_{q_{i}}^{i}\left(q_{i}, q_{j}\right)\left(I_{i}-\delta q_{i}\right)+V_{q_{j}}^{i}\left(\phi_{j}\left(q_{i}, q_{j}\right)-\delta q_{j}\right)
\end{array}\right\} .
$$

Maximisation of the right-hand-side yields $V_{q_{i}}^{i}=\gamma I_{i}+\varphi q_{i}$, which after substitution gives

$$
I_{i}=\phi_{i}\left(q_{i}, q_{j}\right)=\frac{\alpha_{1}+\left(\alpha_{3}-\varphi\right) q_{i}+\alpha_{5} q_{j}}{\gamma}
$$

Similarly, we obtain

$$
I_{j}=\phi_{j}\left(q_{i}, q_{j}\right)=\frac{\alpha_{1}+\left(\alpha_{3}-\varphi\right) q_{j}+\alpha_{5} q_{i}}{\gamma}
$$

Substituting $I_{i}=\phi_{i}\left(q_{i}, q_{j}\right), I_{j}=\phi_{j}\left(q_{i}, q_{j}\right), V_{q_{i}}^{i}\left(q_{i}, q_{j}\right)=\alpha_{1}+\alpha_{3} q_{i}+\alpha_{5} q_{j}, V_{q_{j}}^{j}\left(q_{i}, q_{j}\right)=\alpha_{1}+$ $\alpha_{3} q_{j}+\alpha_{5} q_{i}, V_{q_{j}}^{i}=\alpha_{2}+\alpha_{4} q_{j}+\alpha_{5} q_{i}$ into the (HJB) equation, we obtain

$$
\rho V^{i}\left(q_{i}, q_{j}\right)=\left\{\begin{array}{c}
\left(T+\frac{p-c}{2}\right)+\frac{p-c}{2 \tau} q_{i}-\frac{p-c}{2 \tau} q_{j}-\frac{\gamma}{2}\left(\frac{\alpha_{1}+\left(\alpha_{3}-\varphi\right) q_{i}+\alpha_{5} q_{j}}{\gamma}\right)^{2} \\
-\frac{\beta}{2} q_{i}^{2}-\varphi q_{i}\left(\frac{\alpha_{1}+\left(\alpha_{3}-\varphi\right) q_{i}+\alpha_{5} q_{j}}{\gamma}\right) \\
+\left(\alpha_{1}+\alpha_{3} q_{i}+\alpha_{5} q_{j}\right)\left(\left(\frac{\alpha_{1}+\left(\alpha_{3}-\varphi\right) q_{i}+\alpha_{5} q_{j}}{\gamma}\right)-\delta q_{i}\right) \\
+\left(\alpha_{2}+\alpha_{4} q_{j}+\alpha_{5} q_{i}\right)\left(\frac{\alpha_{1}+\left(\alpha_{3}-\varphi\right) q_{j}+\alpha_{5} q_{i}}{\gamma}-\delta q_{j}\right)
\end{array}\right\}
$$


which can be rewritten as

$$
\begin{aligned}
& \left(\rho \alpha_{0}-T-\frac{p-c}{2}-\frac{1}{2 \gamma} \alpha_{1}^{2}-\frac{1}{\gamma} \alpha_{1} \alpha_{2}\right) \\
& +q_{i}\left(\alpha_{1}(\rho+\delta)-\frac{p-c}{2 \tau}-\frac{\alpha_{1} \alpha_{3}}{\gamma}-\frac{\alpha_{2} \alpha_{5}}{\gamma}-\frac{\alpha_{1} \alpha_{5}}{\gamma}+\frac{\alpha_{1} \varphi}{\gamma}\right) \\
& +q_{j}\left(\alpha_{2}(\rho+\delta)+\frac{p-c}{2 \tau}-\frac{\alpha_{2} \alpha_{3}}{\gamma}-\frac{\alpha_{1} \alpha_{4}}{\gamma}-\frac{\alpha_{1} \alpha_{5}}{\gamma}+\frac{\alpha_{2} \varphi}{\gamma}\right) \\
& +q_{i}^{2}\left(\alpha_{3}\left(\frac{\rho}{2}+\delta\right)-\frac{\alpha_{3}^{2}}{2 \gamma}-\frac{\alpha_{5}^{2}}{\gamma}+\frac{\beta}{2}+\frac{\varphi \alpha_{3}}{\gamma}-\frac{\varphi^{2}}{2 \gamma}\right) \\
& +q_{j}^{2}\left(\alpha_{4}\left(\frac{\rho}{2}+\delta\right)-\frac{\alpha_{3} \alpha_{4}}{\gamma}-\frac{\alpha_{5}^{2}}{2 \gamma}+\frac{\varphi \alpha_{4}}{\gamma}\right) \\
& +q_{i} q_{j}\left(\alpha_{5}\left((\rho+2 \delta)-\frac{2 \alpha_{3}}{\gamma}-\frac{\alpha}{\gamma}+\frac{2 \varphi}{\gamma}\right)\right)=0 .
\end{aligned}
$$

For the equality to hold, the terms in brackets in the above equation have to be equal to zero. From the last line $\alpha_{5}=0$, which also implies $\alpha_{4}=0$ from the second-last line, so that the above simplifies to

$$
\begin{aligned}
& \left(\rho \alpha_{0}-T-\frac{p-c}{2}-\frac{\alpha_{1}^{2}}{2 \gamma}-\frac{\alpha_{1} \alpha_{2}}{\gamma}\right) \\
& +q_{i}\left(\alpha_{1}(\rho+\delta)-\frac{p-c}{2 \tau}-\frac{\alpha_{1} \alpha_{3}}{\gamma}+\frac{\alpha_{1} \varphi}{\gamma}\right) \\
& +q_{j}\left(\alpha_{2}(\rho+\delta)+\frac{p-c}{2 \tau}-\frac{\alpha_{2} \alpha_{3}}{\gamma}+\frac{\alpha_{2} \varphi}{\gamma}\right) \\
& +q_{i}^{2}\left(\alpha_{3}\left(\frac{\rho}{2}+\delta+\frac{\varphi}{\gamma}\right)-\frac{\alpha_{3}^{2}}{2 \gamma}+\frac{\beta}{2}-\frac{\varphi^{2}}{2 \gamma}\right)=0 .
\end{aligned}
$$

Solving from last equation for $\alpha_{3}$, we obtain

$$
\alpha_{3}=\varphi+\gamma\left(\frac{\rho}{2}+\delta\right) \mp \frac{1}{2} \sqrt{4 \gamma(\beta+\rho \varphi+2 \delta \varphi)+\gamma^{2}(\rho+2 \delta)^{2}}
$$

We choose the negative root, since $V_{q_{i} q_{i}}^{i}=\alpha_{3}<0$ needs to be satisfied for the maximisation problem to be concave. We therefore focus on:

$$
\alpha_{3}=\varphi+\gamma\left(\frac{\rho}{2}+\delta\right)-\sqrt{\gamma(\beta+\rho \varphi+2 \delta \varphi)+\gamma^{2}\left(\frac{\rho}{2}+\delta\right)^{2}}
$$


which substituted in the second equation gives

$$
\alpha_{1}=\frac{p-c}{\tau} \frac{1}{\rho+\sqrt{4 \frac{\beta+\rho \varphi+2 \delta \varphi}{\gamma}+(\rho+2 \delta)^{2}}} .
$$

Recall that $I_{i}=\frac{\alpha_{1}+\left(\alpha_{3}-\varphi\right) q_{i}+\alpha_{5} q_{j}}{\gamma}=\frac{\alpha_{1}+\left(\alpha_{3}-\varphi\right) q_{i}}{\gamma}$. Since in the steady state $I_{i}=\delta q_{i}$, we obtain that

$$
I_{C L}^{s}=\frac{\alpha_{1}}{\gamma} \frac{1}{\left(1-\frac{\left(\alpha_{3}-\varphi\right)}{\delta \gamma}\right)},
$$

which after substituting for $\alpha_{1}$ and $\alpha_{3}$ gives

$$
I_{C L}^{s}=\frac{(p-c) \delta}{2 \tau[\beta+\gamma \delta(\delta+\rho)+\varphi(2 \delta+\rho)]}
$$

which coincides with the steady state open-loop solution, as given by (22) in Section 3. 


\section{Appendix B. Closed-loop solution with increasing marginal cost}

The purchaser's instantaneous objective function is:

$$
T+p\left(\frac{1}{2}+\frac{q_{i}-q_{j}}{2 \tau}\right)-\frac{c}{2}\left(\frac{1}{2}+\frac{q_{i}-q_{j}}{2 \tau}\right)^{2}-\frac{\gamma}{2} I_{i}^{2}-\frac{\beta}{2} q_{i}^{2} .
$$

Define the value function as

$$
V^{i}\left(q_{i}, q_{j}\right)=\alpha_{0}+\alpha_{1} q_{i}+\alpha_{2} q_{j}+\left(\alpha_{3} / 2\right) q_{i}^{2}+\left(\alpha_{4} / 2\right) q_{j}^{2}+\alpha_{5} q_{i} q_{j}
$$

The value function has to satisfy the HJB equation:

$$
\rho V^{i}\left(q_{i}, q_{j}\right)=\max \left\{\begin{array}{c}
\left(T+\frac{p}{2}-\frac{c}{8}\right)+\left(\frac{2 p-c}{4 \tau}\right) q_{i}-\left(\frac{2 p-c}{4 \tau}\right) q_{j} \\
-\left(\frac{c}{8 \tau^{2}}+\frac{\beta}{2}\right) q_{i}^{2}-\frac{c}{8 \tau^{2}} q_{j}^{2}+\frac{c}{4 \tau^{2}} q_{i} q_{j}-\frac{\gamma}{2} I_{i}^{2} \\
+V_{q_{i}}^{i}\left(q_{i}, q_{j}\right)\left(I_{i}-\delta q_{i}\right)+V_{q_{j}}^{i}\left(\phi_{j}\left(q_{i}, q_{j}\right)-\delta q_{j}\right)
\end{array}\right\}
$$

Maximisation of the RHS yields

$$
\begin{aligned}
I_{i} & =\phi_{i}\left(q_{i}, q_{j}\right)=\frac{1}{\gamma} V_{q_{i}}^{i}\left(q_{i}, q_{j}\right)=\frac{\alpha_{1}+\alpha_{3} q_{i}+\alpha_{5} q_{j}}{\gamma}, \\
I_{j} & =\phi_{j}\left(q_{i}, q_{j}\right)=\frac{1}{\gamma} V_{q_{j}}^{j}\left(q_{i}, q_{j}\right)=\frac{\alpha_{1}+\alpha_{3} q_{j}+\alpha_{5} q_{i}}{\gamma}, \\
V_{q_{j}}^{i} & =\alpha_{2}+\alpha_{4} q_{j}+\alpha_{5} q_{i} .
\end{aligned}
$$

After substitution, we obtain

$$
\rho V^{i}\left(q_{i}, q_{j}\right)=\left\{\begin{array}{c}
\left(T+\frac{p}{2}-\frac{c}{8}\right)+\left(\frac{2 p-c}{4 \tau}\right) q_{i}-\left(\frac{2 p-c}{4 \tau}\right) q_{j} \\
-\left(\frac{c}{8 \tau^{2}}+\frac{\beta}{2}\right) q_{i}^{2}-\frac{c}{8 \tau^{2}} q_{j}^{2}+\frac{c}{4 \tau^{2}} q_{i} q_{j}-\frac{\gamma}{2} I_{i}^{2} \\
+V_{q_{i}}^{i}\left(\frac{1}{\gamma} V_{q_{i}}^{i}-\delta q_{i}\right) \\
+V_{q_{j}}^{i}\left(\phi_{j}\left(q_{i}, q_{j}\right)-\delta q_{j}\right)
\end{array}\right\}
$$


which provides

$$
\rho V^{i}\left(q_{i}, q_{j}\right)=\left\{\begin{array}{c}
\left(T+\frac{p}{2}-\frac{c}{8}\right)+\left(\frac{2 p-c}{4 \tau}\right) q_{i}-\left(\frac{2 p-c}{4 \tau}\right) q_{j} \\
-\left(\frac{c}{8 \tau^{2}}+\frac{\beta}{2}\right) q_{i}^{2}-\frac{c}{8 \tau^{2}} q_{j}^{2}+\frac{c}{4 \tau^{2}} q_{i} q_{j} \\
-\frac{1}{2 \gamma}\left(V_{q_{i}}^{i}\right)^{2}+\frac{1}{\gamma}\left(V_{q_{i}}^{i}\right)^{2}-\delta q_{i} V_{q_{i}}^{i} \\
+\frac{1}{\gamma} V_{q_{j}}^{j} V_{q_{j}}^{i}-\delta q_{j} V_{q_{j}}^{i}
\end{array}\right\}
$$

or

$$
\rho V^{i}\left(q_{i}, q_{j}\right)=\left\{\begin{array}{c}
\left(T+\frac{p}{2}-\frac{c}{8}\right)+\left(\frac{2 p-c}{4 \tau}\right) q_{i}-\left(\frac{2 p-c}{4 \tau}\right) q_{j} \\
-\left(\frac{c}{8 \tau^{2}}+\frac{\beta}{2}\right) q_{i}^{2}-\frac{c}{8 \tau^{2}} q_{j}^{2}+\frac{c}{4 \tau^{2}} q_{i} q_{j} \\
+\frac{1}{2 \gamma}\left(V_{q_{i}}^{i}\right)^{2}-\delta q_{i} V_{q_{i}}^{i}+\frac{1}{\gamma} V_{q_{j}}^{j} V_{q_{j}}^{i}-\delta q_{j} V_{q_{j}}^{i}
\end{array}\right\}
$$

Recall:

$$
\begin{aligned}
V_{q_{i}}^{i}\left(q_{i}, q_{j}\right) & =\alpha_{1}+\alpha_{3} q_{i}+\alpha_{5} q_{j}, \\
V_{q_{j}}^{j}\left(q_{i}, q_{j}\right) & =\alpha_{1}+\alpha_{3} q_{j}+\alpha_{5} q_{i}, \\
V_{q_{j}}^{i} & =\alpha_{2}+\alpha_{4} q_{j}+\alpha_{5} q_{i} .
\end{aligned}
$$

Substituting, we obtain

$$
\rho V^{i}\left(q_{i}, q_{j}\right)=\left\{\begin{array}{c}
\left(T+\frac{p}{2}-\frac{c}{8}\right)+\left(\frac{2 p-c}{4 \tau}\right) q_{i}-\left(\frac{2 p-c}{4 \tau}\right) q_{j} \\
-\left(\frac{c}{8 \tau^{2}}+\frac{\beta}{2}\right) q_{i}^{2}-\frac{c}{8 \tau^{2}} q_{j}^{2}+\frac{c}{4 \tau^{2}} q_{i} q_{j} \\
+\frac{1}{2 \gamma}\left(\alpha_{1}+\alpha_{3} q_{i}+\alpha_{5} q_{j}\right)^{2}-\delta q_{i}\left(\alpha_{1}+\alpha_{3} q_{i}+\alpha_{5} q_{j}\right) \\
+\frac{1}{\gamma}\left(\alpha_{1}+\alpha_{3} q_{j}+\alpha_{5} q_{i}\right)\left(\alpha_{2}+\alpha_{4} q_{j}+\alpha_{5} q_{i}\right)-\delta q_{j}\left(\alpha_{2}+\alpha_{4} q_{j}+\alpha_{5} q_{i}\right)
\end{array}\right\}
$$


For the equality to hold, the following terms in brackets have to be equal to zero:

$$
\begin{aligned}
& \left(\rho \alpha_{0}-T+\frac{c}{8}-\frac{p}{2}-\frac{\alpha_{1}^{2}}{2 \gamma}-\frac{\alpha_{1} \alpha_{2}}{\gamma}\right) \\
& +q_{i}\left(\alpha_{1}(\rho+\delta)-\frac{2 p-c}{4 \tau}-\frac{\alpha_{1} \alpha_{3}}{\gamma}-\frac{\alpha_{2} \alpha_{5}}{\gamma}-\frac{\alpha_{1} \alpha_{5}}{\gamma}\right) \\
& +q_{j}\left(\alpha_{2}(\rho+\delta)+\frac{2 p-c}{4 \tau}-\frac{\alpha_{2} \alpha_{3}}{\gamma}-\frac{\alpha_{1} \alpha_{4}}{\gamma}-\frac{\alpha_{1} \alpha_{5}}{\gamma}\right) \\
& +q_{i}^{2}\left(\alpha_{3}\left(\frac{\rho}{2}+\delta\right)+\frac{c}{8 \tau^{2}}-\frac{\alpha_{3}^{2}}{2 \gamma}-\frac{\alpha_{5}^{2}}{\gamma}+\frac{\beta}{2}\right) \\
& +q_{j}^{2}\left(\alpha_{4}\left(\frac{\rho}{2}+\delta\right)+\frac{c}{8 \tau^{2}}-\frac{\alpha_{3} \alpha_{4}}{\gamma}-\frac{\alpha_{5}^{2}}{2 \gamma}\right) \\
& +q_{i} q_{j}\left(\alpha_{5}(2 \delta+\rho)-\frac{2}{\gamma} \alpha_{3} \alpha_{5}-\frac{\alpha_{4} \alpha_{5}}{\gamma}-\frac{c}{4 \tau^{2}}\right)=0
\end{aligned}
$$

Solving the last three equations in $\alpha_{3}, \alpha_{4}$ and $\alpha_{5}$, we obtain two candidates for solution:

$$
\begin{gathered}
\alpha_{1}=\frac{\left(p-\frac{c}{2}\right)\left(\alpha_{5}-\gamma \rho\right)}{4 \tau[\beta+\gamma \delta(\rho+\gamma \delta)]}, \\
\alpha_{2}=\frac{(2 p-c)}{4 \tau[\beta+\gamma \delta(\rho+\gamma \delta)]}\left[\frac{1}{2} \rho \gamma-\frac{\left(c+40 \tau^{2} \beta+10 \tau^{2} \gamma(\rho+2 \delta)^{2}\right) \alpha_{5}}{4\left(c+4 \tau^{2} \beta+\tau^{2} \gamma(\rho+2 \delta)^{2}\right)}\right] \\
\alpha_{3}=\gamma\left(\frac{\rho}{2}+\delta\right)-\frac{\alpha_{5}}{2} \\
\alpha_{4}=-\frac{\left(5 c-16 \tau^{2} \beta-4 \tau^{2} \gamma(\rho+2 \delta)^{2}\right) \alpha_{5}}{\left(4 c+16 \tau^{2} \beta+4 \tau^{2} \gamma(\rho+2 \delta)^{2}\right)}
\end{gathered}
$$

and

$$
\alpha_{5}=-\frac{\gamma}{3} \sqrt{\frac{1}{\gamma}\left(\frac{c}{\tau^{2}}+4 \beta\right)+(\rho+2 \delta)^{2}}<0
$$

or

$$
\alpha_{5}=\frac{\gamma}{3} \sqrt{\frac{1}{\gamma}\left(\frac{c}{\tau^{2}}+4 \beta\right)+(\rho+2 \delta)^{2}}>0 .
$$

The choice of the correct root among the two alternatives for $\alpha_{5}-(\mathrm{B} 19)$ and (B20) - is made by noting that the negative root, (B19), implies $\alpha_{3}>0$, which makes the value function convex in quality. Thus, we disregard this solution and conclude that the positive root, (B20), is the correct 
one. To obtain the steady state values of quality and investment, recall that $I_{i}=\frac{\alpha_{1}+\alpha_{3} q_{i}+\alpha_{5} q_{j}}{\gamma}$. In the steady state $q_{i}=I_{i} / \delta$, which implies $I_{C L}^{s}=\frac{\alpha_{1}}{\gamma-\frac{1}{\delta}\left(\alpha_{3}+\alpha_{5}\right)}$ or, after substitution,

$$
I_{C L}^{s}=\left(\frac{\rho-\frac{\alpha_{5}}{\gamma}}{\rho+\frac{\alpha_{5}}{\gamma}}\right) \frac{(2 p-c) \delta}{4 \tau[\beta+\gamma \delta(\rho+\gamma \delta)]},
$$

where $\alpha_{5}$ is given by (B20). Investment is positive if $\rho \gamma>\alpha_{5}$. However, from (B17) we see that this condition is never fulfilled when $\alpha_{3}<0$. Thus, the steady state equilibrium is a corner solution with $I_{C L}^{s}=0$. 
Figure 1. Equilibrium is a saddle point

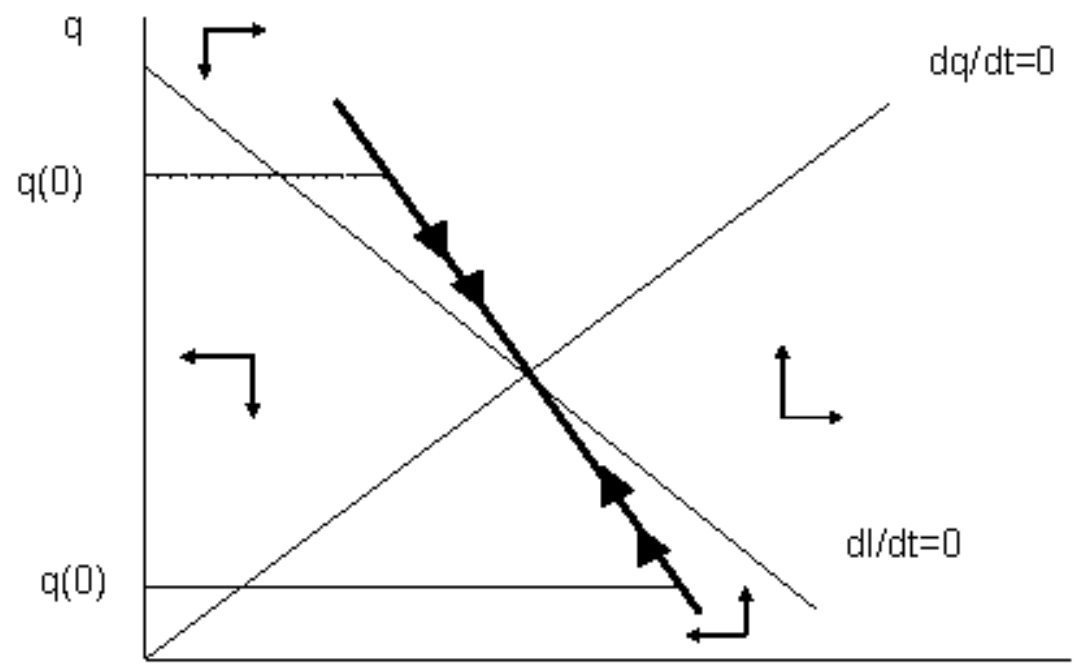

Figure 2. Increase in price or reduction in travel costs (more competition)

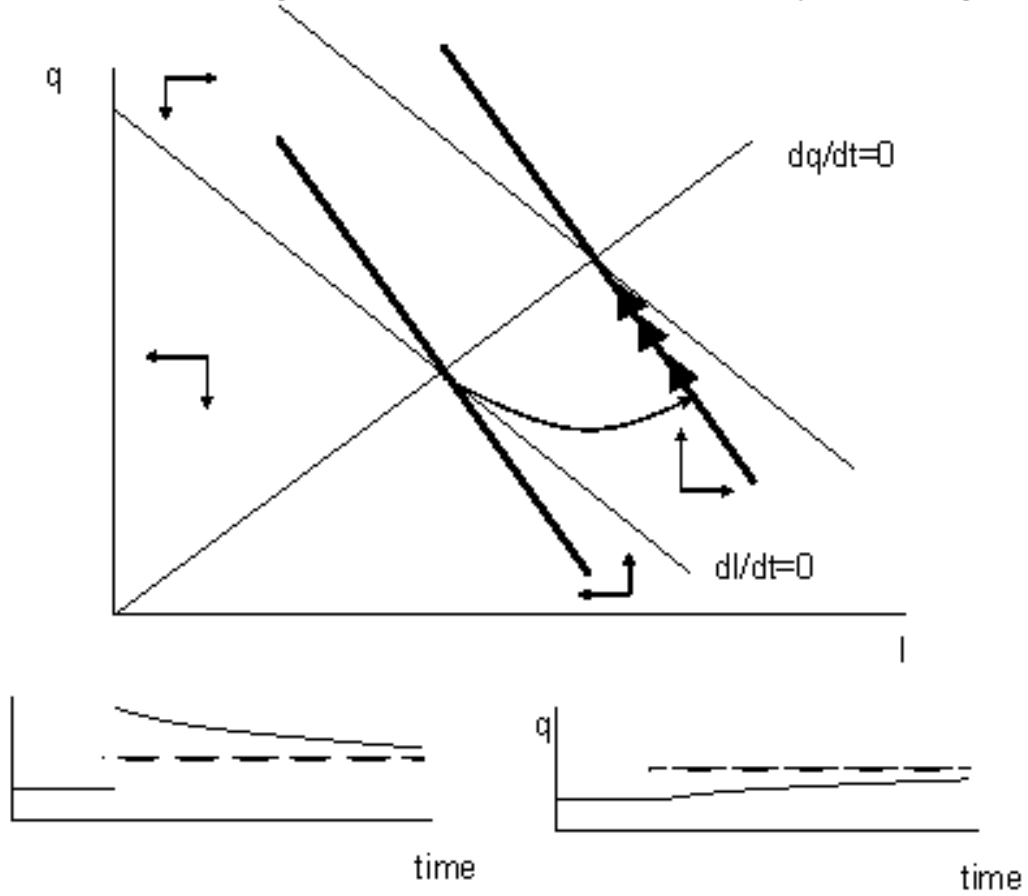

